\title{
UNIFORM APPROXIMATION BY SOLUTIONS OF ELLIPTIC EQUATIONS
}

\author{
BARNET M. WEINSTOCK ${ }^{1}$
}

\begin{abstract}
The space $H_{A}(K)$ of continuous functions on a compact set $K$ in Euclidean space which can be uniformly approximated by solutions of the elliptic, constant-coefficient partial differential equation $A f=0$ is studied. In particular, it is shown that $H_{A}(K)$ is local, in the same sense as in the theory of rational approximation in the complex plane. Simultaneous approximation of functions and their derivatives is also considered.
\end{abstract}

Let $K$ be a compact set in $\AA^{n}$ and let $A$ be a partial differential operator on $R^{n}$. We denote by $H_{A}(K)$ the uniform closure on $K$ of the space of functions satisfying the equation $A f=0$ in some neighborhood of $K$. The properties of $H_{A}(K)$ when $n=2$ and $A=\partial / \partial x+i \partial / \partial y$ are well known. (See, for example, Chapter 3 of [1].) One of the more interesting of these properties is that $H_{A}(K)$ is local in the sense that a continuous function $f$ belongs to $H_{A}(K)$ if each $x \in K$ has a compact neighborhood $N$ such that $f \in H_{A}(K \cap N)$.

Kallin [6] has shown that $H_{A}(K)$ is not local in general if $K$ is a compact set in $C^{m}$ and $A=\bar{\partial}$, the Cauchy-Riemann operator. (See [7], however, where $H_{\tilde{\delta}}(K)$ is shown to be local for certain product sets in $C^{m}$.) The purpose of this note is to present some results concerning $H_{A}(K)$ when $A$ is an elliptic operator with constant coefficients, in particular the result that $H_{A}(K)$ is local. Simultaneous uniform approximation of functions and their derivatives by solutions of $A f=0$ is also considered.

Many of these results are contained explicitly or implicitly in the work of F. Browder ([2], [3]) who considered the case of elliptic equations with variable coefficients. However, the simplicity of the proofs in the constant coefficient case when contrasted with the elaborate techniques from functional analysis required in the general situation seems to warrant separate treatment.

Let $A$ be a linear partial differential operator on $\boldsymbol{R}^{n}$ with constant coefficients. A fundamental solution for $A$ is a distribution $E$ on $\boldsymbol{R}^{n}$ such

Received by the editors October 18, 1972 and, in revised form, February 2, 1973. AMS (MOS) subject classifications (1970). Primary 35J30, 46E15; Secondary 41A30, 35E99.

Key words and phrases. Uniform approximation, elliptic partial differential equations.

${ }^{1}$ This work was partially supported by an NSF Grant at Brown University.

(c) American Mathematical Society 1973 
that $A E=\delta$, where $\delta$ is the unit point mass at 0 . In particular, $A E=0$ on $\boldsymbol{R}^{n}-\{0\}$. Suppose now that $A$ is elliptic of order $m$ with real coefficients. (In this case $m$ is necessarily even.) If $E$ is a fundamental solution for $A$ then $E \mid \boldsymbol{R}^{n}-\{0\} \in C^{\infty}\left(\boldsymbol{R}^{n}-\{0\}\right)$ and

$$
\begin{aligned}
E(x) & =O\left(|x|^{m-n}\right), & & n \text { odd, } \\
& =O\left(|x|^{m-n} \log |x|\right), & & n \text { even. }
\end{aligned}
$$

(See, for example, [5].) Thus we can state the following result:

Proposition 1. If $A$. is an elliptic operator of order $m$ on $\boldsymbol{R}^{n}$, with constant real coefficients, then every fundamental solution $E$ for $A$ satisfies $D^{\alpha} E \in L_{\mathrm{loc}}^{1}\left(\boldsymbol{R}^{n}\right)$ for $|\alpha| \leqq m-1$.

Henceforth, $A$ will denote an operator satisfying the hypotheses of Proposition 1 . We note that the formal adjoint $A^{\prime}$ also satisfies these conditions.

Proposition 2. Let $K$ be a compact set in $\boldsymbol{R}^{n}$ and let $T$ be a distribution of order $k \leqq m-1$ with support in $K$. If $T(f)=0$ for all $f \in C^{\infty}\left(\boldsymbol{R}^{n}\right)$ such that $A f=0$ in a neighborhood of $K$ then there exists $F \in L^{1}$ with support in $K$ such that $D^{\alpha} F \in L^{1}$ for $|\alpha| \leqq m-k-1$ and such that $A^{\prime} F=T$.

Proof. Let $F=T * E$, Then $F$ is a distribution satisfying $A^{\prime} F=T$. If $\Delta$ is a closed ball disjoint from $K$ and $\phi$ is a $C^{\infty}$ function supported in $\Delta$ then $f(x)=\int_{\Delta} E(y-x) \phi(y) d y$ is a $C^{\infty}$ function satisfying $A f=0$ in a neighborhood of $K$. Hence $T(f)=0$. But $T(f)=(T * E)(\phi)$. Thus $F$ is supported in $K$. Finally, $T=\sum_{|q| \leqq k} D^{q} \mu_{q}$ where each $\mu_{q}$ is a measure with compact support in $\boldsymbol{R}^{n}$. Hence,

$$
F=T * E=\sum_{|q| \leqq k} \mu_{q} * D^{\alpha} E,
$$

so Proposition 1 implies that $D^{\alpha} F$ is locally integrable if $|\alpha| \leqq m-k-1$.

If $K$ is a compact subset of $\boldsymbol{R}^{n}$ let $C^{k}(K), k=0,1,2, \cdots$, denote the space of germs of $C^{k}$ functions on $K$, i.e., the space of equivalence classes of functions of class $C^{k}$ in a neighborhood of $K$ where two functions are considered equivalent if their derivatives up to order $k$ agree on $K$. We make $C^{k}(K)$ into a normed space by setting

$$
\|f\|_{k}=\max _{|\alpha| \leqq k} \max _{x \in K}\left|D^{\alpha} f(x)\right| .
$$

If $U$ is a neighborhood of $K$ each function $f$ in $C^{k}(U)$ defines an element of $C^{k}(K)$ which we will denote again by $f$. It is clear that every continuous linear functional on $C^{k}(K)$ can be identified with a distribution of order $\leqq k$ whose support lies in $K$. 
Let $H_{A}^{k}(K)$ denote the closure in $C^{k}(K)$ of (the germs on $K$ of ) the functions $f$ satisfying $A f=0$ in some neighborhood of $K$.

Proposition 3. Let $K$ be a compact set of Lebesgue measure zero in $\boldsymbol{R}^{n}$. Then $H_{A}^{k}(K)=C^{k}(K)$ for $0 \leqq k \leqq m-1$.

Proof. By the above remarks it suffices to show that if $T$ is a distribution of order $\leqq m-1$ with support in $K$ such that $T(f)=0$ for all $f$ satisfying $A f=0$ in some neighborhood of $K$ then $T=0$. But, by Proposition 2, $T=A^{\prime} F$, where $F$ is an integrable function supported in $K$. Since $K$ has measure $0, F$ vanishes, hence $T=0$.

For general compact sets $K$ we have the following approximation theorem:

Proposition 4. Let $U$ be a neighborhood of $K$ and let $f \in C^{m}(U)$. If $A f=0$ on $K$ then $f \in H_{A}^{k}(K), k=0, \cdots, m-1$.

Proof. Again, it suffices to show that every distribution $T$ of order $\leqq m-1$ with support in $K$ which satisfies $T(g)=0$ for all $g$ satisfying $A g=0$ in some neighborhood of $K$ also annihilates $f$. But $T=A^{\prime} F$, where $F$ is a measure supported on $K$, hence $T(f)=\left(A^{\prime} F\right)(f)=F(A f)=0$, since $A f=0$ on $K$.

The next result shows that membership in $H_{A}^{k}(K)$ is a local property in a rather strong sense, for $0 \leqq k \leqq m-1$.

Proposition 5. If $f \in C^{k}(K)$ and if for each $x \in K$ there is a neighborhood $U_{x}$ of $x$ in $R^{n}$ such that $f \in H_{A}^{k}\left(K \cap \bar{U}_{x}\right)$ then $f \in H_{A}^{k}(K)$.

Proposition 5 follows immediately from the following result:

Proposition 6. Let T be a distribution of order $\leqq m-1$ with support in $K$ such that $T(f)=0$ for all $f$ satisfying $A f=0$ in some neighborhood of $K$. If $\left\{U_{i}\right\}$ is a finite open covering of $K$ there exist distributions $T_{i}$ of order $\leqq m-1$ such that $\sum T_{i}=T, T_{i}$ is supported in $K \cap \bar{U}_{i}$, and $T_{i}(f)=0$ for all $f$ satisfying $A f=0$ in a neighborhood of $K \cap \bar{U}_{i}$.

Proof. Let $\left\{\phi_{i}\right\}$ be a smooth partition of unity subordinate to the covering $\left\{U_{i}\right\}$. Then, by Proposition 2,

$$
T=A^{\prime} F=A^{\prime} \sum \phi_{i} F=\sum A^{\prime}\left(\phi_{i} F\right) .
$$

Let $T_{i}=A^{\prime}\left(\phi_{i} F\right)$. Then $T_{i}$ is a distribution supported in $K \cap \bar{U}_{i}, \sum T_{i}=T$, and if $A f=0$ in a neighborhood of $K \cap U_{i}$ then $T_{i}(f)=\left(\phi_{i} F\right)(A f)=0$. It remains to show that $T_{i}$ has order $\leqq m-1$. But $T_{i}=A^{\prime} \phi_{i} F=\phi_{i} A^{\prime} F+R$ where $R$ is a sum of terms involving derivatives of order $\leqq m-1$ of $F$. 
Let $B_{A}^{k}(K)$ denote the subspace of $C^{k}(K)$ consisting of functions satisfying $A f=0$ in the interior of $K$. As an application of Proposition 5 we can show that $B_{A}^{k}(K)=H_{A}^{k}(K)$ if $K$ is sufficiently well behaved.

A set $K$ is said to have the segment property if for each $x \in K$ there is a neighborhood $U$ of $x$ and a vector $w_{x}$ such that, if $0<t<1$ and $z \in K \cap U$ then $z+t w_{x}$ lies in the interior of $K$. If $K$ is the closure of an open set $D$ whose boundary is a hypersurface of class $C^{1}$ then $K$ has the segment property.

Proposition 7. Let $K$ have the segment property. Then $B_{A}^{k}(K)=$ $H_{A}^{k}(K), 0 \leqq k \leqq m-1$.

P.ROOF. By Proposition 5 it suffices to show that if $f \in C^{k}(K)$ and $A f=0$ on int $K$, then in a neighborhood of each point $x$ of $K$ the function $f$ can be approximated by functions $f_{n}$ satisfying $A f_{n}=0$ locally. But if $K$ has the segment property we can choose $f_{n}$ to be a suitable translate of $f$ in the direction $w_{x}$, since translation is continuous in the $C^{k}$ topology and $A$ commutes with translations.

It is of interest to note that instead of approximation by germs, i.e., by functions satisfying $A f=0$ in an arbitrary neighborhood of $K$ as in Propositions 3, 4, or 7, one may require that the approximating functions satisfy $A f=0$ on any fixed neighborhood $U$ of $K$ such $U-K$ is connected. This follows from the general theory of linear partial differential operators with constant coefficients, in particular from Theorem 3.4.3 of [4] together with the fact that solutions of homogeneous elliptic equations are analytic.

Finally, we consider briefly the case of nonelliptic operators with constant coefficients.

Let $C^{\infty}(K)$ be the space of germs on $K$ of $C^{\infty}$ functions defined in neighborhoods of $K$. We give $C^{\infty}(K)$ the topology determined by the seminorms $\left\{\|f\|_{k}: k=0,1,2, \cdots\right\}$ defined above. We denote by $H_{A}^{\infty}(K)$ the closure in $C^{\infty}(K)$ of the functions satisfying $A f=0$ in some neighborhood of $K$.

An open set $U \subset \boldsymbol{R}^{n}$ is called $A$-convex if $A C^{\infty}(U)=C^{\infty}(U)$ (cf. [4, Chapter 3]). If $A$ is elliptic then every open $U$ is $A$-convex (and conversely).

Proposition 2'. Let $K$ be a compact set which is the intersection of a decreasing sequence of $A$-convex open sets. If $T$ is a distribution with support in $K$ and if $T(f)=0$ for all $f \in C^{\infty}\left(R^{n}\right)$ such that $A f=0$ in a neighborhood of $K$ then there exists a distribution $S$ with support in $K$ such that $A^{\prime} S=T$.

Proof. Let $K=\bigcap \Omega_{j}$, where $\Omega_{j+1} \subset \Omega_{j}$ and each $\Omega_{j}$ is an $A$-convex open set. For each $j$ we can regard $T$ as an element of the dual of the Fréchet space $C^{\infty}\left(\Omega_{j}\right)$. As such it is orthogonal to the kernel of the continuous operator 
$A$ which maps $C^{\infty}\left(\Omega_{j}\right)$ onto $C^{\infty}\left(\Omega_{j}\right)$. Hence there is a distribution $S_{j}$ with compact support in $\Omega_{j}$ such that $A^{\prime} S_{j}=T$. But $A^{\prime}$ is one-to-one on distributions with compact support, so there is a distribution $S$, with compact support in $\boldsymbol{R}^{n}$ such that $S_{j}=S$ for all $j$. Clearly $S$ is supported in $K$ and $A^{\prime} S=T$.

Using Proposition $2^{\prime}$ one can derive analogues of Propositions 5 and 7 for the spaces $H_{A}^{\infty}(K)$ and $B_{A}^{\infty}(K)$. In particular, one has the following result:

PROPOSITION 8. Let $K$ be the closure of a domain in $R^{n}$ with $C^{\infty}$ boundary. If $K$ is the intersection of a sequence of $A$-convex open sets then for each $f \in C^{\infty}(K)$ such that $A f=0$ on the interior of $K$ there is a sequence $f_{n}$ of $C^{\infty}$ functions, each satisfying $A f_{n}=0$ on some neighborhood of $K$, such that $\left\|f-f_{n}\right\|_{k} \rightarrow 0$ for all $k$.

\section{REFERENCES}

1. A. Browder, Introduction to function algebras, Benjamin, New York, 1969. MR 39 \#7431.

2. F. Browder, Approximation by solutions of partial differential equations, Amer. J. Math. 84 (1962), 134-160. MR 31 \#2505.

3. - Functional analysis and partial differential equations. II, Math. Ann. 145 (1961/62), 81-226. MR 25 \#318.

4. L. Hörmander, Linear partial differential operators, Die Grundlehren der math. Wissenschaften, Band 116, Academic Press, New York; Springer-Verlag, Berlin, 1963. MR 28 \#4221.

5. F. John, Plane waves and spherical means applied to partial differential equations, Interscience Tracts in Pure and Appl. Math., vol. 2, Interscience, New York, 1955. MR 17, 746.

6. E. Kallin, A non-local function algebra, Proc. Nat. Acad. Sci. U.S.A. 49 (1963), 821-824. MR 27 \#2878.

7. B. Weinstock, Approximation by holomorphic functions on certain product sets in $C^{n}$, Pacific J. Math. 43 (1972), 811-822.

Department of Mathematics, Brown University, Providence, Rhode Island 02912

Current address: Department of Mathematics, University of Kentucky, Lexington, Kentucky 40506 